\title{
Prekallikrein Deficiency Presenting as Recurrent Cerebrovascular Accident: Case Report and Review of the Literature
}

\author{
Esteban Uribe Bojanini, Arturo Loaiza-Bonilla, and Agustin Pimentel \\ Division of Hematology and Department of Medicine, Sylvester Comprehensive Cancer Center, \\ University of Miami Miller School of Medicine, 1475 NW 12th Ave Suite 3300, Miami, FL 33136, USA \\ Correspondence should be addressed to Arturo Loaiza-Bonilla, aloaizabonilla@med.miami.edu
}

Received 12 June 2012; Accepted 24 July 2012

Academic Editors: S. Aref, E. Arellano-Rodrigo, and K. Konstantopoulos

Copyright (C 2012 Esteban Uribe Bojanini et al. This is an open access article distributed under the Creative Commons Attribution License, which permits unrestricted use, distribution, and reproduction in any medium, provided the original work is properly cited.

\begin{abstract}
We report the case of a woman with history of hypertension and hyperlipidemia presenting with recurrent episodes consistent clinically with cerebrovascular accidents (CVA), and MRI changes suggestive of ischemia versus vasculitis as their cause. No anatomical neurological, rheumatic, cardioembolic, or arteriosclerotic etiologies could be determined by extensive workup. Incidentally, the patient was found to have prolonged activated Partial Thromboplastin Time (aPTT) and a normal Prothrombin Time (PT); further testing revealed a prekallikrein deficiency. Since no other cause for the CVAs was established, and other prothrombotic states were ruled out, it is proposed that they are clinical manifestations derived from the prekallikrein deficiency, which in a patient with known cardiovascular risk factors could lead to thrombotic complications such as stroke.
\end{abstract}

\section{Case Presentation}

A 32-year-old African American woman presented to the neurology clinic for followup, after having 4 episodes of cerebrovascular accidents (CVA) in the last 4 years. The cause for the CVA episodes had not been established despite extensive studies, including magnetic resonance imaging (MRI), echocardiogram with bubble study, CT angiography, carotid Doppler ultrasound, Holter monitoring, and after implementing secondary prevention measures with aspirin, statin, and blood pressure control. All of the episodes were associated with sudden left-sided weakness and dysarthria, lasting up to 2 hours in the first three episodes and 2 weeks in the last one, but they were self-limited, with complete resolution of symptoms afterwards. Due to the unclear diagnosis an MRI was ordered, which revealed high FLAIR and diffusion-weighted imaging (DWI) signal surrounding the frontal horns of the lateral ventricle; this was suggestive of an ischemic etiology versus vasculitis. The patient was admitted to the hospital for complete workup, seeking a definite diagnosis.

The patient had a prior medical history of hypertension treated with verapamil, and dyslipidemia treated with simvastatin, and she was taking aspirin $81 \mathrm{mg}$ daily since her first episode of CVA. No history of oral or transdermal contraception. Review of systems revealed no history of bleeding or other thrombotic events in the past. She denied prolonged bleeding after dental procedures, menometrorrhagia, or pregnancy losses. No past surgical history. She denied alcohol, tobacco abuse, or illicit drug abuse. Her family and social history were noncontributory.

On admission, her physical examination showed a normotensive, afebrile patient, with no signs of bleeding or thrombosis, neurological symptoms, or focal deficits. Her initial complete blood count and comprehensive metabolic panel were revealed within normal limits.

Evaluation of coagulation times revealed a prolonged aPTT of $99.4 \mathrm{sec}$, with a normal PT of $11 \mathrm{sec}$. She was not on 
any anticoagulation therapy. Mixing studies were ord-ered. The aPTT was corrected to $30.1 \mathrm{sec}$ after a $1: 1 \mathrm{mix}$ with normal plasma, indicating a coagulation factor deficiency and excluding the presence of an inhibitor of the intrinsic system. The activities of factors VIII, IX, XI, XII, and Von Willebrand factor (vWF) were in the normal range. Fibrinogen was normal and lupus anticoagulant assays were negative. Platelet func-tion assays were normal. The abnormal aPTT shortened with prolonged incubation. Following these results, a deficit in the contact phase of the intrinsic coagulation pathway was suspected, more particularly a prekallikrein deficiency. A prekallikrein (PK) activity level was ordered, which revealed a $1 \%$ activity (on both clotting and amidolytic activity assays). PK antigen level was undetectable, which led to the final diagnosis of prekallikrein deficiency.

In order to rule out other potential, concomitant prothrombotic states, several tests were ordered, including Protein C, Protein S, Antithrombin III, Beta 2 Microglobulin, Anticardiolipin antibodies, lupus anticoagulant, CD55/CD59 per flow cytometry, factor V Leyden, Jak-2, and Prothrombin gene 202010 mutation analyses. All of these were unremarkable. Homocysteine levels were normal, and Sickle Cell Screen was also negative.

To rule out vasculitis or any other rheumatoid disease, a panel was ordered including antinuclear antibodies, antiSSA, anti-SSB, anti-Smith, anti-RNP, antiscleroderma-70, antiribosomal antibody, antidouble-stranded DNA, antineutrophil cytoplasmic antibodies, rheumatoid factor, and complement C3 and C4, which were all normal.

A 12-lead electrocardiogram revealed normal sinus rhythm with no arrhythmias. Magnetic resonance carotid and vertebrobasilar angiogram revealed no arterial flow defects or atherosclerotic disease. The patient was not considered a candidate for thrombolysis or systemic anticoagulation due to the fact that it was not clear whether it was an acute or chronic event.

After completion of the aforementioned diagnostic workup, the patient was discharged home with diagnosis of prekallikrein deficiency. She was recommended to continue close surveillance by neurology and hematology, and to continue on daily aspirin and her hypertension and dyslipidemia medications.

\section{Discussion}

Prekallikrein $(\mathrm{PK})$ is a glycoprotein synthesized in the liver and secreted as a single-chain peptide with a molecular weight of 88,000 Daltons and a normal plasma concentration of approximately $40 \mathrm{mg} / \mathrm{mL}[1,2]$. More than $75 \%$ of prekallikrein circulates as a complex with high-molecularweight kininogen (HMWK). Only about $2-5 \%$ is free. PK together with Hageman factor (factor XII) and HMWK constitute the contact phase of the intrinsic coagulation and fibrinolytic pathways [3]. Its activation leads to the formation of factor XIIa, factor XIa, and kallikrein (KK) [4]. KK is implicated in many physiological and pathological processes including blood coagulation, the initiation of the classical complement cascade pathway, and the formation of bradykinin and renin [5].

Prekallikrein deficiency is a rare autosomal recessive disease, which was first described in 1965 by Hathaway et al. [6] in a family with 4 of 11 siblings showing a prolonged aPTT, caused by a deficiency of an unknown factor, which he called "Fletcher factor." In 1973 Wuepper et al. [7] determined that the "Fletcher factor" was prekallikrein. Chromosomal localization of the gene has been mapped to the q34-q35 region on the long arm of chromosome 4 , encoded by a single gene (KLKB1), and composed of 15 exons and 14 introns [8]. Seven types of mutations in prekallikrein deficient families have been identified by molecular genetic analysis, with most mutations being found in the light chain region of the prekallikrein gene [9].

Only around 80 cases of this disease have been reported in the literature, due to the fact that most of the patients are asymptomatic; it is likely that a large number of individuals go unrecognized and unreported [10]. Clinical manifestations are not clearly defined, as most of the patients remain asymptomatic and are diagnosed after an incidental finding of prolonged aPTT and a normal PT [11]. While PK, HMWK, and factor XII are required for a normal aPTT, they do not appear to be essential for normal coagulation. Despite the presence of a prolonged aPTT, patients with PK deficiency have no bleeding tendency, mostly due to the fact that proteins of the contact system are not key elements of the coagulation pathway, and they rather play a secondary role in the generation of thrombin. It has been reported that such factors deficiencies do not result in a marked bleeding diathesis, as most of the factor XI activation that is preferentially mediated via platelets and thrombin $[11,12]$.

Other coagulation factors deficiencies have been reported to present occasionally with thrombotic events [13]. These events have been reported in hemophilia and von Willebrand disease (VWD), but also in rare conditions like fibrinogen or factor VII defects [14]. Only patients deficient in factor II or factor $\mathrm{X}$ have not been reported with thrombosis [15]. It is still unclear whether defects in the contact phase components of blood coagulation, including PK-which are not associated with bleeding diathesis-represent a mild prothrombotic state or not.

Recent studies have suggested that factor XII deficiency is not a prothrombotic condition since most of the patients who were reported with thrombosis also had other acquired or congenital known risk factors [16, 17].

Several reports of thrombotic events in patients diagnosed with prekallikrein deficiency have been published. In 1983, Goodnough et al. [18] reviewed all 37 patients that had been diagnosed with PK deficiency and found 4 cases of thrombosis (10.8\%). In 2010, Girolami et al. [10] reviewed the total of 75 patients reported with the deficiency, and found 9 thrombotic events $(12.2 \%)$, which is a very similar incidence between the 2 reviews. Girolami et al. also reported associated prothrombotic risk factors in 6 of the 9 cases. They concluded that it is certain that PK deficiency does not protect from thrombosis in spite of the marked in vitro clotting defect, but a clear association with prothrombotic state cannot be made [10]. A limitation of these studies 
was that many patients were not studied for congenital prothrombotic defects.

\section{Conclusion}

In this paper we report the case of a woman with history of hypertension and hyperlipidemia presenting with recurrent episodes consistent clinically with cerebrovascular accidents (CVA), and MRI changes suggestive of ischemia versus vasculitis as their cause. It is relevant that the patient had previous diagnosis of hypertension and dyslipidemia, as they are known risk factors for stroke. No anatomical neurological, rheumatic, cardioembolic, or arteriosclerotic etiologies could be determined by extensive workup. Incidentally, the patient was found to have prolonged activated Partial Thromboplastin Time (aPTT) and a normal Prothrombin Time (PT); further testing revealed a prekallikrein deficiency. It is still unclear whether PK deficiency can be associated with prothrombotic state or not, and the rarity and underreporting of such cases make a prospective study a difficult task. In this particular case, since no other cause for the CVAs was established in our patient, and other prothrombotic states were ruled out, it is proposed that they are clinical manifestations derived from the prekallikrein deficiency, which in a patient with known cardiovascular risk factors could lead to thrombotic complications such as stroke. The infrequency with which PK is encountered and its pathophysiology make it a formidable diagnostic and therapeutic challenge.

\section{References}

[1] A. Girolami, P. Scarparo, N. Candeo, and A. M. Lombardi, "Congenital prekallikrein deficiency," Expert Review of Hematology, vol. 3, no. 6, pp. 685-695, 2010.

[2] A. Girolami, S. Marun, S. Vettore et al., "A large family from Argentina with Prekallikrein deficiency due to a compound heterozygosis ( $\mathrm{T}$ insertion in intron 7 and Asp558Glu in exon 15): prekallikrein Cordoba," American Journal of Hematology, vol. 85, no. 5, pp. 363-366, 2010.

[3] H. L. Meier, J. V. Pierce, R. W. Colman, and A. P. Kaplan, "Activation and function of human Hageman factor. The role of high molecular weight kininogen and prekallikrein," Journal of Clinical Investigation, vol. 60, no. 1, pp. 18-31, 1977.

[4] L. M. Asmis, I. Sulzer, M. Furlan, and B. Lämmle, "Prekallikrein deficiency: the characteristic normalization of the severely prolonged aPTT following increased preincubation time is due to autoactivation of factor XII," Thrombosis Research, vol. 105, no. 6, pp. 463-470, 2002.

[5] J. Tang, C. L. Yu, S. R. Williams et al., "Expression, crystallization, and three-dimensional structure of the catalytic domain of human plasma kallikrein," Journal of Biological Chemistry, vol. 280, no. 49, pp. 41077-41089, 2005.

[6] W. E. Hathaway, L. P. Belhasen, and H. S. Hathaway, "Evidence for a new plasma thromboplastin factor. I. Case report, coagulation studies and physicochemical properties," Blood, vol. 26, no. 5, pp. 521-532, 1965.

[7] K. D. Wuepper, "Prekallikrein deficiency in man," Journal of Experimental Medicine, vol. 138, no. 6, pp. 1345-1355, 1973.
[8] J. W. Bryant and Z. Shariat-Madar, "Human plasma kallikreinkinin system: physiological and biochemical parameters," Cardiovascular and Hematological Agents in Medicinal Chemistry, vol. 7, no. 3, pp. 234-250, 2009.

[9] T. Nakao, T. Yamane, T. Katagami et al., "Severe prekallikrein deficiency due to a homozygous Trp499Stop nonsense mutation," Blood Coagulation and Fibrinolysis, vol. 22, no. 4, pp. 337-339, 2011.

[10] A. Girolami, E. Allemand, I. Bertozzi, N. Candeo, S. Marun, and B. Girolami, "Thrombotic events in patients with congenital prekallikrein deficiency: a critical evaluation of all reported cases," Acta Haematologica, vol. 123, no. 4, pp. 210-214, 2010.

[11] K. Acar, M. Yağci, G. T. Sucak, and R. Haznedar, "Isolated prolonged activated partial thromboplastin time in an asymptomatic patient: fletcher factor deficiency," Thrombosis Research, vol. 118, no. 6, pp. 765-766, 2006.

[12] C. S. Kitchens, "The contact system," Archives of Pathology and Laboratory Medicine, vol. 126, no. 11, pp. 1382-1386, 2002.

[13] M. Franchini, "Thrombotic complications in patients with hereditary bleeding disorders," Thrombosis and Haemostasis, vol. 92, no. 2, pp. 298-304, 2004.

[14] A. Girolami, E. Ruzzon, F. Tezza, R. Scandellari, S. Vettore, and B. Girolami, "Arterial and venous thrombosis in rare congenital bleeding disorders: a critical review," Haemophilia, vol. 12, no. 4, pp. 345-351, 2006.

[15] A. Girolami, N. Candeo, S. Vettore, A. M. Lombardi, and B. Girolami, "The clinical significance of the lack of arterial or venous thrombosis in patients with congenital prothrombin or FX deficiency," Journal of Thrombosis and Thrombolysis, vol. 29, no. 3, pp. 299-302, 2010.

[16] A. Girolami, M. L. Randi, S. Gavasso, A. M. Lombardi, and F. Spiezia, "The occasional venous thromboses seen in patients with severe (Homozygous) FXII deficiency are probably due to associated risk factors: a study of prevalence in 21 patients and review of the literature," Journal of Thrombosis and Thrombolysis, vol. 17, no. 2, pp. 139-143, 2004.

[17] A. Girolami, N. Candeo, G. B. De Marinis, E. Bonamigo, and B. Girolami, "Comparative incidence of thrombosis in reported cases of deficiencies of factors of the contact phase of blood coagulation," Journal of Thrombosis and Thrombolysis, vol. 31, no. 1, pp. 57-63, 2011.

[18] L. T. Goodnough, H. Saito, and O. D. Ratnoff, "Thrombosis or myocardial infarction in congenital clotting factor abnormalities and chronic thrombocytopenias: a report of 21 patients and a review of 50 previously reported cases," Medicine, vol. 62, no. 4, pp. 248-255, 1983. 


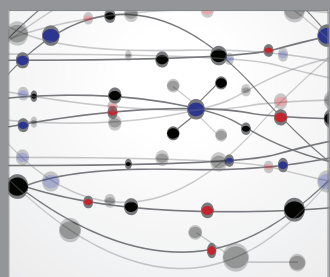

The Scientific World Journal
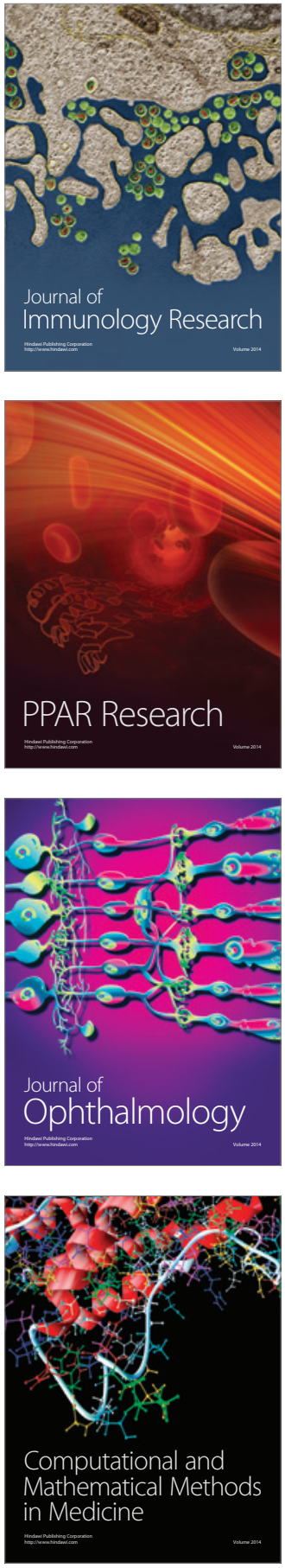

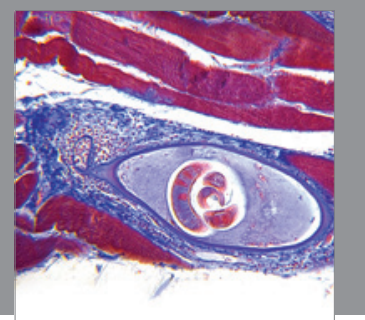

Gastroenterology

Research and Practice
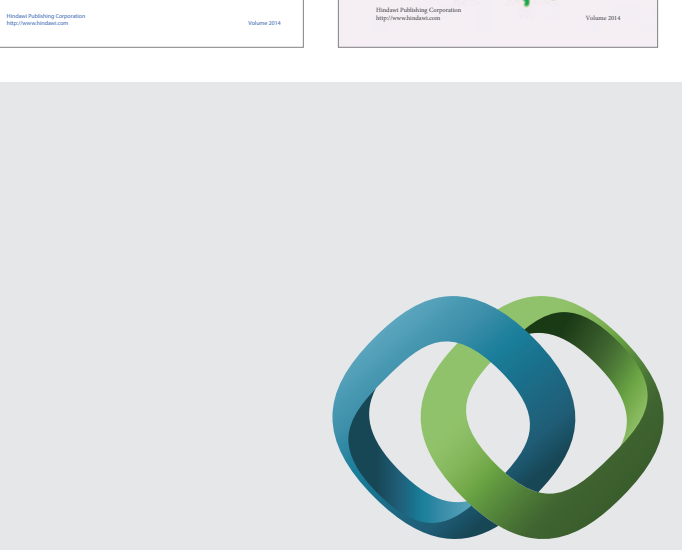

\section{Hindawi}

Submit your manuscripts at

http://www.hindawi.com
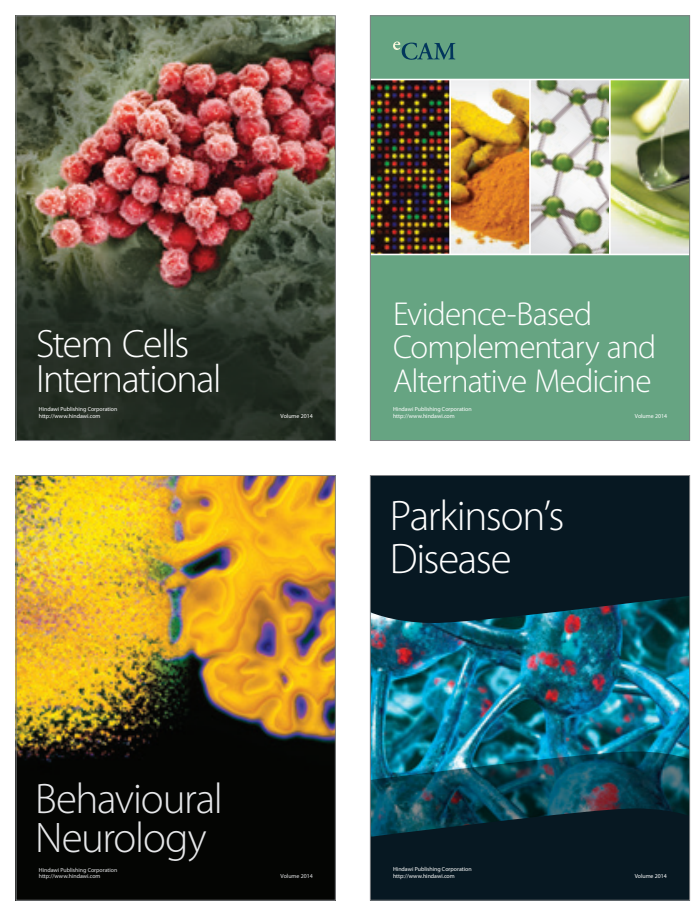

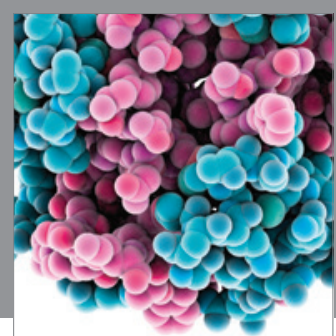

Journal of
Diabetes Research

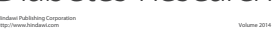

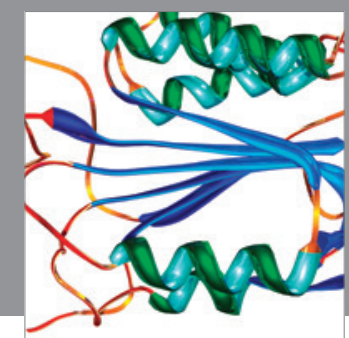

Disease Markers
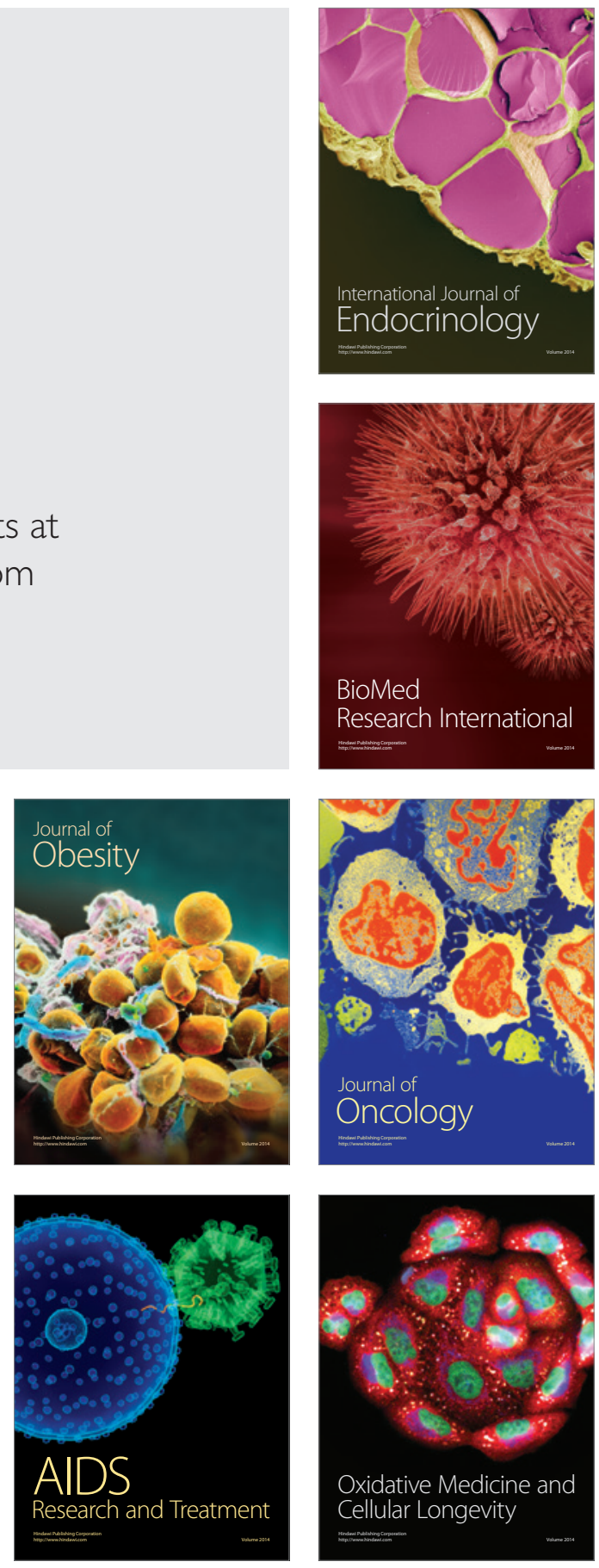